\title{
Research and Improvement of Airport Security System
}

\author{
Yinhuan Li \\ School of North China Electric Power University, Baoding 071000, China. \\ 18730217961@163.com
}

\begin{abstract}
In view of the fact that the airport security system may result in the long time of security check and the bad experience for passengers, we put forward the security optimization model based on Queuing Theory. First of all, we look for problems in the entire security system. We deal with the known data and establish the queuing theory $\mathrm{M} / \mathrm{M} / 1$ model according to the airport security queuing theory through the flow chart of the security system analysis. We use the Analytic Hierarchy Process to analyze the influence of the different weight of different factors in the airport security system based on the data and access to information, we can find the problem areas that affect the security system therefore. Secondly, we conducted a correlation analysis of the common problems in the process of queuing in the airport and advise the airport security department on how to effectively improve the passenger throughput of the airport security system and reduce the waiting time of passengers. In conclusion, we set up a security system to find problems in the area of the relevant mechanisms and put forward the optimization strategy for each problem in this paper. Then we have put forward practical and feasible improvement measures especially for the passenger security system with different cultural differences.
\end{abstract}

Keywords: Security queuing theory; analytic hierarchy process; cultural difference.

\section{Introduction}

In modern society, the aircraft has the advantages of short path, fast speed and so on, it has become more and more people choose to travel transport. But we all know, before the plane, we have to spend a long waiting time. In order to ensure flight safety, we have to go through layers of security checks, for our trip brings inconvenient. In China, because the rapid development of high-speed rail, coupled with a series of cumbersome procedures before boarding the plane, more people will choose to take the high-speed rail, the airline is undoubtedly a great loss.

In the United States, there are many passengers are not satisfied with the security check before boarding, and even decided to complain to the airline. So, to optimize the entire security process for the interests of airlines and passengers flying experience is very important.

\section{Model Hypothesis}

Do not consider the cost of passengers on the passport, visa procedures.

Two aircraft cannot take off at the same time, the shortest interval of 3 minutes. (according to the statistics of the airline company)

The data show that the aircraft's guest rate is generally around $80 \%$, where to take the number of $80 \%$.

\section{Queuing Network Model}

Queuing system in modern airport security system is a kind of normal, we get the physical model based on the queuing phenomenon and further establish the queuing theory of mathematical model.

The queuing theory model, given the following input information (probability distribution): arrival rate $\lambda$, Business Hours $T$, or attendant service rate $\mu$, output

information (average): q, W. It is assumed that the arrival time of the customer is Poisson distribution, and the service time of the server obeys exponential distribution. A typical queuing model $\mathrm{M} / \mathrm{M} / 1$ model is obtained [1]:

Average number of customers in the system: 


$$
q=E[N]=\frac{\rho}{(1-\rho)}
$$

Average waiting time of customers in the system:

$$
T_{q}=\frac{1}{\mu(1-\rho)}
$$

Service utilization rate:

$$
\rho=\frac{\lambda}{\mu}
$$

The data are processed by an airport system:

Table 1. Average time, variance and service rate of each process

\begin{tabular}{|c|c|c|c|}
\hline Process & The average time & Variance & $\mu$ (Service Rate) \\
\hline ID Check Process & $10.21 \mathrm{~s}, 2.6 \mathrm{~s}$ & $20.53,8.88$ & 5 passengers $/ \mathrm{min}$ \\
\hline X-Ray Scan & $3.7 \mathrm{~s}, 7.5 \mathrm{~s}$ & $11.64,66.76$ & 10 passengers $/ \mathrm{min}$ \\
\hline Millimeter Wave Scan & $11.6 \mathrm{~s}$ & 34.36 & 5 passengers $/ \mathrm{min}$ \\
\hline To get scanned property & $28 \mathrm{~s}$ & 198.53 & 3 passengers $/ \mathrm{min}$ \\
\hline
\end{tabular}

So, we can get the following formula:

$$
L_{q}=\rho L_{x}=\frac{\lambda}{\mu-\lambda} \times \frac{\lambda}{\mu}=\frac{\lambda^{2}}{\mu(\mu-\lambda)}
$$

Combined with the analysis of Table 1, we can see that the average queue length is the biggest, so it is easy to form the bottleneck. According to the model analysis, it can be seen that the service efficiency of the airport security inspection system has an important relationship with the waiting time and waiting queue. The actual airport security system bottlenecks can be judged according to the length of the queue and the number of security checks.

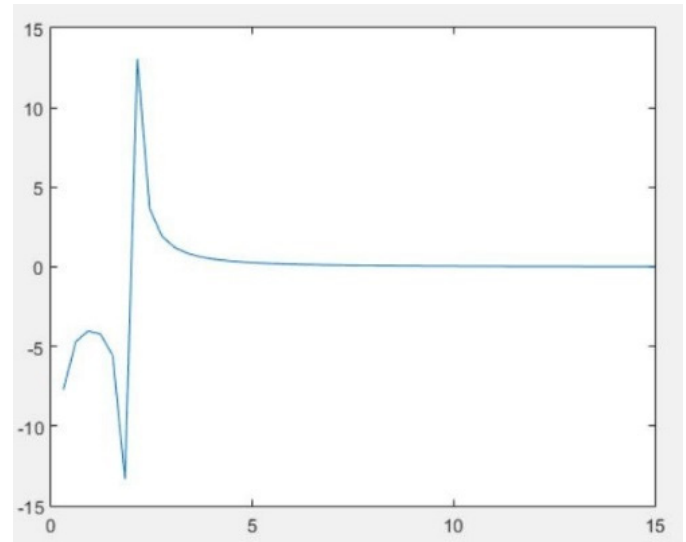

Figure 1. The relationship between queue length and service rate

\section{Analytic Hierarchy Process [2]}

According to the data obtained and the data obtained, we can evaluate the work ability of the security system and establish a hierarchy analysis model. The level of the model includes:

Target level ------ the ability to measure the security system;

Criteria layer ------machine condition (A1), staff working ability (A2), emergency situation (A3).

Solution layer------ID detection (B1), millimeter wave Scanner Detection (B2), x ray screening (B3).

The structure contrast matrix is [3]: 


$$
\begin{aligned}
& A_{0}=\left[\begin{array}{lll}
1.000 & 2.000 & 0.333 \\
0.500 & 1.000 & 0.142 \\
3.000 & 7.000 & 1.000
\end{array}\right] \quad B_{1}=\left[\begin{array}{lll}
1.000 & 0.333 & 0.500 \\
3.000 & 1.000 & 0.605 \\
2.000 & 1.651 & 1.000
\end{array}\right] \\
& B_{2}=\left[\begin{array}{lll}
1.000 & 5.000 & 5.000 \\
0.200 & 1.000 & 0.500 \\
0.200 & 2.000 & 1.000
\end{array}\right] \quad B_{3}=\left[\begin{array}{lll}
1.000 & 0.410 & 0.248 \\
2.437 & 1.000 & 0.605 \\
4.026 & 1.651 & 1.000
\end{array}\right]
\end{aligned}
$$

Simultaneous equations, we get:

$$
Z=0.20043 B_{1}+0.35041 B_{2}+0.44916 B_{3}
$$

According to the weights can be easily learned that the priority of the investigation of the area of the problem: item detection $>$ X-ray $>$ Millimeter wave $>$ ID check.

So, when the queue is too long and the excessive number of site security should be in accordance with article item detection, X-ray, Millimeter wave, ID check to carry on the investigation, it can be timelier and accurately find the problem areas, reduce the waiting time of passengers.

\section{The Solution to the Problem}

Queuing problem can be regarded as an ideal queuing theory model.

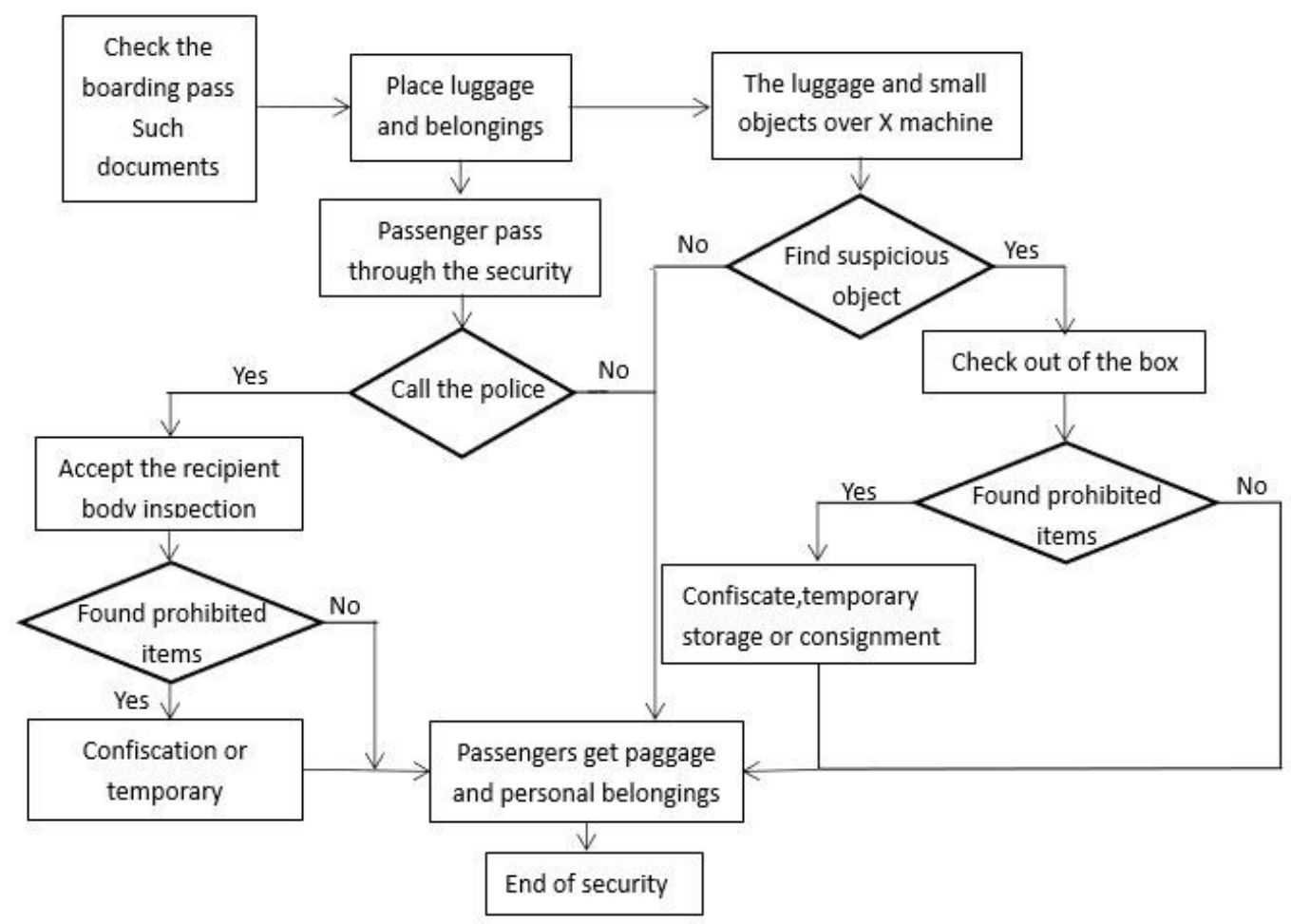

Figure 2. The specific process of queuing security inspection

\section{Summary}

According to the time index of each link used in the screening process and access to information, influence on various aspects of the overall security system by AHP, determine a certain amount of weight, and has certain accuracy in checking relevant information.

Some of the strategies we have developed can effectively alleviate the problem of large passenger throughput and long waiting time. 
Based on the analysis of the influence of cultural differences on the passenger screening process, we developed a strategy, in real life settings has certain guiding significance.

We have limited data and available data, so the model may not be universal.

We qualitatively analyze the subjective factors in some problems, which may also influence the relevant solving process.

\section{References}

[1]. Li Birchen, Su Gulping. Study on the bottleneck problem of [J]. Microelectronics and computer database system based on queuing network model, 2012, (06): 100-103.

[2]. Yu Shingle. Mathematical modeling classic case actual combat of [J]. Case study of mathematical modelling, 2015, (1): 202- 208.

[3]. Zeng Junkie. Airport security setting and optimization of [J]. Knowledge economy, 2009, (12): 173-174. 